\section{Intersections}

Canadian Journal of Music

Revue canadienne de musique
Intersections CANADIAN JOURAL OF MUSIC
REVUE CANADIENEE DE MUSIOUH

\title{
Une approche contextualiste des relations voix/gestes dans les improvisations de Tanya Tagaq : un " acte performanciel "
}

\section{Sophie Stévance}

Volume 36, numéro 2, 2016

URI : https://id.erudit.org/iderudit/1051598ar

DOI : https://doi.org/10.7202/1051598ar

Aller au sommaire du numéro

\section{Éditeur(s)}

Canadian University Music Society / Société de musique des universités canadiennes

ISSN

1911-0146 (imprimé)

1918-512X (numérique)

Découvrir la revue

Citer cet article

Stévance, S. (2016). Une approche contextualiste des relations voix/gestes dans les improvisations de Tanya Tagaq : un « acte performanciel ». Intersections, 36(2), 57-66. https://doi.org/10.7202/1051598ar
Résumé de l'article

La pratique artistique de Tanya Tagaq se caractérise par le recours à de nombreux effets vocaux, issus tant du katajjaq que d'autres traditions musicales, que la chanteuse semble coordonner à des mouvements et des gestes expressifs. L'hypothèse est que les mouvements du bras, des mains, du corps de Tagaq sur scène possèdent, tout comme les sons qu'elle produit, une dimension symbolique pertinente pour la performance et se coordonnent dans une même expression. Peut-on identifier une typologie de correspondances voix/gestes lors d'une performance de Tagaq? Pour explorer son geste musical en rapport avec la production du son, les données ont été générées par la captation des mouvements et de la voix lors de performances scéniques (LARC et Palais Montcalm, Québec, janvier-février 2016), à partir d'un système de motion capture (VICON), d'un microphone de gorge et d'un traitement informatique. On explorera ici un terrain symbolique, éclaté, souvent inaccessible car métaphorique, et peu foulé par la recherche, notamment du fait de la singularité de Tagaq qui intègre autant des éléments de sa culture inuit que ceux de la culture transnationale. Étant donné sa signification expressive pour l'auditoire, il s'agit de saisir ce que pourrait signifier ou exprimer le mouvement corporel en lien avec les émissions vocales de l'artiste.
Copyright ( C Canadian University Music Society / Société de musique des universités canadiennes, 2018
Ce document est protégé par la loi sur le droit d'auteur. L’utilisation des services d'Érudit (y compris la reproduction) est assujettie à sa politique d'utilisation que vous pouvez consulter en ligne.

https://apropos.erudit.org/fr/usagers/politique-dutilisation/ 


\section{UNE APPROCHE CONTEXTUALISTE DES RELATIONS VOIX/GESTES DANS LES IMPROVISATIONS DE TANYA TAGAQ: UN "ACTE PERFORMANCIEL"}

\section{Sophie Stévance}

Tanya Tagaq est une chanteuse inuite qui pratique une forme de chant de gorge traditionnel (le katajjaq) dans un contexte de musique pop-électronique-expérimental. Alors que dans le katajjaq traditionnel, deux femmes sont placées face à face, très proches l'une de l'autre, et émettent à tour de rôle des sons sur un rythme rapide et soutenu qui seront imités ou complétés par l'autre, Tanya Tagaq le pratique seule, parfois accompagnée par des musiciens. Cela dit, l'artiste maîtrise la technique traditionnelle du jeu de gorge et en connaît les codes symboliques, tout comme elle maîtrise ceux de la scène musicale pop. Ouverte à l'exploration, Tanya Tagaq s'approprie les codes de ces deux cultures dans lesquelles elle puise ses influences. Comment utilise-t-elle les images, les symboles reliés à son patrimoine d'origine?

L'ensemble de sa pratique artistique fait appel au corps, à la sensualité, à l'humain, au vivant. On ne peut, par exemple, pas s'en tenir au sens des paroles de ses chansons: "j'ai beaucoup trop d'idées et d'opinions politiques, voilà pourquoi je n'écris pas beaucoup de textes - rarement pour ainsi dire:j'aurais bien trop de choses à dire" ${ }^{1 !}$ ». Tanya Tagaq ne considère pas le langage comme un véhicule premier pour sa musique, même si elle utilise de manière sporadique quelques mots au sein de ses improvisations. Par contre, dans ses performances improvisées, son utilisation du corps est frappante: les mouvements du bras, des mains, des doigts, du corps de l'artiste sur scène semblent posséder, tout comme les sons qu'elle produit, une dimension symbolique pertinente pour la performance, et semblent se coordonner dans une même expression. Mais quelle est la nature de ces rapports voix-gestes? Nul doute que le corps fait partie intégrante de l'art de Tagaq. D’ailleurs, l'évocation par le corps et la voix de son patrimoine est éloquente dans le documentaire auquel elle a participé

1 Tanya Tagaq, entretien avec Sophie Stévance, 8 septembre 2013, Laboratoire audionumérique de recherche et de création (LARC), Université Laval. 
en $2009^{2}$. Partant de ce fait, son corps pourrait être une composante essentielle des performances de l'artiste, autant que ne l'est la production vocale.

Lors d'un premier entretien d'autoconfrontation croisée avec Tanya Tagaq, en février 2016, où il était question de visionner ensemble quelques-uns de ses propres gestes corporels codés avec le mannequin $3 \mathrm{D}$ VICON, elle affirmait reconnaître notamment un ours et un aigle. Elle cherche, selon elle, par son corps à exprimer son imaginaire inuit, ce qui l'habite. Bien qu'il n'y ait pas nécessairement de relation entre le geste musical et la production du son (Zbikowski 2011), le geste signifie ou exprime quelque chose, même inconsciemment, en raison de sa signification expressive pour l'auditoire ainsi que pour l'artiste. Dans de précédentes recherches, nous avons analysé les «fonctions» de la voix de Tagaq au sein de ses improvisations, et la manière dont la chanteuse construit ses improvisations sur la base du katajjaq traditionnel (Stévance 2015). À présent, il s'agit de mieux comprendre la place de son corps dans ses rapports avec la voix au sein de ses performances. Quelle est la nature de ces rapports corps/voix? Sont-ils co-modaux? corrélés? dépendants? isolés? $\mathrm{Ou}$ au contraire interagissent-ils dans des contextes particuliers? Que représentent-ils? Constituent-ils une "chorégraphie» (au service, par exemple, de la musique?), ou symbolisent-ils quelque chose d'autre, de plus profond?

Depuis 2016, dans le cadre de notre projet de recherche intitulé «Development of an interactive prototype of the improvised musical Performance ${ }^{3}$ " qui réunit plusieurs chercheurs et ingénieurs de l'Université Laval (Sophie Stévance, Serge Lacasse, Denis Laurendeau, avec la collaboration de Denis Ouellet, Sylvain Comtois et Michael Garancher), nous tentons de mieux comprendre la place du corps dans la production musicale de Tagaq en observant les gestes corporels en lien avec les gestes vocaux produits simultanément dans plusieurs de ses performances, que ce soit dans un environnement contrôlé ou sur scène. Plus précisément, le fait d'appréhender la nature de ces rapports permettra de développer des méthodes et des outils en vue de catégoriser ces combinaisons gestes/voix, d'enrichir les méthodes d'observation participante actuelles en combinant approches qualitative et quantitative (Stévance 2017b) tout en investissant le processus de création et de réception et ainsi, à plus long terme, de réaliser une banque de contenus représentatifs de l'univers artistique de Tagaq, lesquels seront transformés en temps réel en fonction à la fois des profils des spectateurs (notamment par la récolte de données biométriques et leur feedback) et de ce qui se déroulera sur scène. Notre objectif est de rehausser l'expérience du spectacle vivant dans une démarche de recherche-création écosophique (Stévance/Lacasse 2015, 2017).

À cette étape-ci de la recherche, les données ont été générées par la captation des gestes corporels et de la voix lors de performances scéniques: au

2 «What We Eat» («tungijuq») avec Tanya Tagaq, réalisé par Zacharias Kunuk : «talk back to Brigitte Bardot and anti-seal hunting lobby on the eternal reality of hunting», 2009. http://www. isuma.tv/fr/tungijuq/tungijuqz2op

3 Ce projet de musicologie/recherche-création bénéficie du soutien du Conseil de recherche en sciences humaines (CRSH) et a été approuvé par le Comité d'éthique de la recherche de l'Université Laval : No d'approbation 2016-309/ 07-06-2017. 
laboratoire audionumérique de recherche et de création (LARC, janvier 2016) et au Palais Montcalm (janvier-février 2016), à partir d'un système de motion capture (VICON), d'un microphone de gorge et d'un traitement informatique. Les premières analyses et codages ont été effectués dès 2014 avec le logiciel Encode développé par le Professeur Francis Dubé4, puis récemment avec le logiciel VNote où les grandes catégories de gestes dégagées en 2014 ont été conservées. À cette étape-ci de l'analyse, il est question d'un travail qualificatif d'observation pour saisir des catégories de gestes corporels en lien avec ses gestes vocaux permettant d'atteindre des degrés de métaphorisation afin de saisir l'agir contextuel de Tanya Tagaq. Cela dit, lors d'un deuxième entretien d'autoconfrontation croisée avec l'artiste, le 18 juin 2017, où il s'agissait de (lui) faire verbaliser les pensées, les intentions, les procédés et tout autre aspect qui a pu déterminer son action sur scène, ainsi que ses perceptions quant aux relations éventuelles entre ses gestes vocaux et son corps, il s'est avéré que les descriptions, simulations, explications et significations présentées par Tagaq corroborent nos analyses.

Exemple 1: https://bit.ly/2CNZaTS

\section{ANALYSE}

En janvier et février 2016, nous avons pu enregistrer Tanya Tagaq en train d'improviser, dans un environnement contrôlé (au LARC), et sur scène, au Palais Montcalm (Ville de Québec) ${ }^{5}$, accompagnant en direct la projection du film Nanook of the North (1922) ${ }^{6}$. Lors de ces deux prestations, Tanya Tagaq portait une combinaison motion-capture (Mocap) ainsi qu'un microphone de gorge.

Exemple 2: http://grecem.oicrm.org/sophie-stevance-une-approche-contextualistedes-relations-voix-gestes-dans-les-improvisations-de-tanya-tagaq-un-acte -performanciel-intersections-canada-150-2018/

Pour mieux comprendre le type de rapport entre les gestes vocaux et corporels de Tanya Tagaq, nous avons appliqué une analyse de type paradigmatique. Prenant appui sur le principe de répétition, ce type d'analyse reste en lien avec le contexte, et donc la narration. De ce point de vue, Tagaq raconte, dans un contexte précis, une histoire - et pas seulement en exécutant la trame sonore du documentaire muet Nanook of the North, mais dans chacune de ses prestations, ainsi qu'elle nous le précisait en juin 2017. En outre, l'analyste, luimême spectateur vivant pleinement l'expérience du spectacle, peut lui aussi se raconter une histoire pour interpréter l'histoire racontée par Tagaq. Ensuite, par répétition, on entend, selon Ruwet, «l'observation d'une identité entre des

4 Concernant l'application Web Encode, voir : $\underline{\text { https://www.youtube.com/watch? } \mathrm{v}=1 \mathrm{TM} 6 \mathrm{bVld}-}$ $\underline{\text { NS8 }}$

5 Nous tenons à remercier l'équipe du Palais Montcalm qui a permis la réalisation de ce projet, notamment Dominique Soutif et Philippe Poulain, ainsi que les productions Six Labels Records, Helen Britton en particulier, qui représentent l'artiste Tanya Tagaq. Merci également aux musiciens œuvrant avec Tanya Tagaq qui ont participé au projet : Jesse Zubot (violon, électronique) et Jean Martin (batterie, électronique) ainsi que Bernard Falaise (guitare) qui s'est joint au trio pour l'occasion.

6 Film/documentaire muet réalisé par Robert Flaherty, 1922 (79') qui met en scène le mode de vie d'une famille inuit à Inukjuaq, situé sur la côte Est de la Baie d'Hudson, Canada. 
segments répartis à divers endroits de la chaîne syntagmatique» (Ruwet 1972, p. 111), c'est-à-dire la succession et la relation entre des unités constituant la phrase. Cela dit, puisque, «du point de vue purement physique, deux évènements concrets ne sont jamais complètement identiques. Une certaine abstraction est donc inévitable (Ruwet, Ibid.), à plus forte raison lorsque l'on se situe dans le domaine de la métaphore, comme c'est le cas dans l'analyse des rapports gestes/voix de la pratique de Tagaq, et même dans la production à proprement parler de l'artiste. On se concentre donc sur les liens de similarités entre des segments apparaissant à deux ou plusieurs reprises, avec ou sans variante, lors du processus de production. Ces segments peuvent être déterminés en repérant, au sein de différentes performances (Nanook of the North au Palais Montcalm, d'autres performances de Nanook of the North, ainsi que des performances individuelles non-thématiques): le nombre de fois où la chanteuse répète une cellule de gestes (à l'identique ou partiellement) et les gestes vocaux produits simultanément; si l'artiste revient à une séquence qui a déjà été énoncée; ou si elle invente une nouvelle cellule, toujours en observant ce que le corps fait simultanément à la production vocale. Il s'agit ensuite d'étudier les traits communs entre ces cellules dans le traitement et le développement de leurs composantes, le renvoi à des unités similaires, ou les contrastes qu'elles affichent entre elles7.

Exemple 3: http://grecem.oicrm.org/sophie-stevance-une-approche-contextualistedes-relations-voix-gestes-dans-les-improvisations-de-tanya-tagaq-un-acte -performanciel-intersections-canada-150-2018/

Exemple 4: enregistrement audiovisuel de Tanya Tagaq au Palais Montcalm, 18 février 2016, et analyse des rapports voix/gestes avec le logiciel VNote, par Michael Garancher http://grecem.oicrm.org/sophie-stevance-une-approche-contextualiste -des-relations-voix-gestes-dans-les-improvisations-de-tanya-tagaq-un-acte-performanciel-intersections-canada-150-2018/

Dans cette vidéo enregistrée au Palais Montcalm le 18 février 2016, on observe des combinaisons de gestes récurrents qui pourraient évoquer des mouvements décrivant, par exemple, des coups de défenses des morses (les gestes vocaux sont alors très graves, comme des grognements), la capture d'un animal lors de la chasse (les gestes vocaux partent alors du très grave au très aigu), le hurlement des canidés (très aigus) ou les représentations onomatopéiques du cri d'oiseaux (comme le pluvier), ou encore la griffe du carcajou (uniquement guttural parlé/grognements). En discutant de ces éléments avec l'artiste, non seulement abonde-t-elle dans le sens de ces descriptions, mais elle nous indique en plus investir l'intérieur de son organisme, en mimant la résection de ses intestins par les voies orales.

Exemple 5: Entretien d'autoconfrontion croisée avec Tanya Tagaq, 18 juin 2017, Laboratoire de recherche-création en musique et multimédia (LARCEM), par Sophie Stévance, Serge Lacasse, Michael Garancher et Francis Dubé http:// grecem.oicrm.org/sophie-stevance-une-approche-contextualiste-des-relations-

7 Une analyse paradigmatique similaire a été appliquée pour l'étude des improvisations musicales vocales de Tanya Tagaq. Voir Stévance 2015. 
voix-gestes-dans-les-improvisations-de-tanya-tagaq-un-acte-performanciel -intersections-canada-150-2018/

Entre toutes ces vidéos, on reconnait des combinaisons de gestes vocaux et corporels récurrents pour évoquer des mouvements qui pourraient décrire, évoquer, son patrimoine inuit, la toundra arctique (son territoire, ses paysages, sa faune). Mais si l'on compare la performance du Palais Montcalm à d'autres vidéos de Nanook of the North ainsi qu'à d'autres performances enregistrées où Tagaq produit des gestes corporels similaires (entre 2014-2016) ${ }^{8}$, on observe notamment, d'une performance à l'autre, que Tagaq ne réalise pas les mêmes gestes, ni corporels ni vocaux. Toutefois, les mêmes ensembles et superpositions décrits pour la performance du Palais Montcalm sont utilisés à d'autres moments de cette même performance, mais également dans d'autres prestations de Nanook of the North de l'artiste ${ }^{9}$. Cette comparaison suggère que son langage se transforme ou interagit avec un contexte en particulier. Une telle constatation a déjà été faite dans nos précédents travaux où il s'agissait d'analyser, sur la base de modélisations, la manière dont Tanya Tagaq construit ses improvisations sur la base du katajjaq traditionnel et de codes culturels et musicaux occidentaux (Stévance 2015). L'étude a démontré que l'artiste utilise des motifs (patterns) musicaux qui reviennent d'une performance à une autre, mais présentés de manière différente; pour autant, ils sont reconnaissables lors de ces expériences distinctes tout en évoquant quelque chose d'autre, aussi en raison des interactions entre l'artiste et les musiciens qui improvisent sur scène avec elle, et les interactions avec le public, lequel change à chaque nouvelle prestation. Ainsi, quel que soit le contexte, il s'agit toujours d'une performance d'improvisation de Tanya Tagaq, dans des circonstances particulières.

\section{UNE ESTHÉTIQUE CONTEXTUALISTE}

Une lecture contextualiste, selon laquelle un phénomène peut s'appréhender en tenant compte de son contexte précis d'évolution, pourrait être un modèle explicatif pertinent, selon nous, pour mieux saisir la nature des rapports gestes/voix dans les performances de Tanya Tagaq. Le contextualisme s'est tout d'abord développé dans le domaine de la philosophie du langage afin de démontrer que la signification des termes varie en fonction du contexte de leur utilisation. Pour le philosophe Levinson (2005), une conception contextualiste envisage qu' "une œuvre d'art est un artefact caractéristique [...] qui est le produit d'une invention humaine, à un moment et à un endroit particuliers, créé par un ou

8 Par exemple : https://www.youtube.com/watch?v=vPAo8mZw5A8, International DRUGA GODBA Festival, 2014; https://www.youtube.com/watch?v=dumvYzfuTow, New York, Metropolitan Museum of Art, 2015; https://www.youtube.com/watch?v=60VE6qlA 3 Do, Tiff Originals, 2016.

9 Voirparexemple:https://www.youtube.com/watch?v=ZG6a1zpJf6A, http://grecem.oicrm.org/ diffusions/tanya-tagaq-culture-ethno-pop/;http://www.youtube.com/watch?v=nuCSckoYsUY; http:// grecem.oicrm.org/diffusions/tanya-tagaq-culture-ethno-pop/; http://www.youtube.com/watch? $\mathrm{v}=$ NxS6gbbJgVw; http://grecem.oicrm.org/diffusions/tanya-tagaq-culture-ethno-pop/; http://www. youtube.com/watch? $\mathrm{v}=699 \mathrm{lzQm} 2 \mathrm{Aeo}$. 
des individus particuliers - ce qui a des conséquences sur la façon dont on fait l'expérience, dont on comprend et évalue correctement les œuvres d'art» (Levinson 2005, p. 127-128). Par conséquent, toute production artefactuelle est ancrée dans son contexte de création et de projection, en dehors duquel cette production cesse d'être ce qu'elle est et ne possède plus les qualités et significations de son contexte originel. Ainsi, selon une lecture contextualiste, la compréhension des rapports gestes/voix dans les performances d'improvisation de Tanya Tagaq réclame une prise en compte des circonstances de leur énonciation. Par conséquent, dans un contexte donné, plusieurs interprétations de ces relations peuvent être autorisées. Cela dit, en tenant compte des contextes de leur présentation, que l'on peut mieux cerner à l'aide d'un dispositif méthodologique adéquat, en particulier l'autoconfrontation croisée $^{10}$, on peut cerner des constantes dans la production vocale et gestuelle de Tagaq, tendant ainsi à révéler des corrélations gestes/voix. L'interprétation est donc teintée par le contexte qui la module. Autrement dit, "Aucune œuvre n'est une île» (Levinson 2005, p. 452): si, pour appréhender l'œuvre de Tagaq, il faut pouvoir considérer, en plus d'analyses comparatives formelles et syntaxiques systématiques (Stévance 2015), son contexte social, historique, culturel et artistique (Stévance 2011), pour saisir les rapports gestes/voix dans les prestations de Tanya Tagaq, il faut aussi tenir compte d'elle-même au moment où elle crée en présence (et avec) d'autrui, donc des énergies variables de ce (ceux) qui l'entourent, musiciens et public, de l'expérience vécue tous ensemble (Stévance 2012).

Les performances de Tanya Tagaq sont incorporées dans le contexte en particulier où elles se déroulent. Ses choix sont établis en fonction, certes, de ce qui la constitue en tant que femme engagée et artiste, mais aussi selon les conventions et horizons qui régissent la performance en train de se dérouler. Ainsi, une analyse rigoureuse ne saurait faire l'économie des procédés de performance, incluant pratique et évènement, qui donnent finalement à la musique toute sa raison d'être. D'ailleurs, à l'instar de la pratique du katajjaq traditionnel (ou de n'importe quelle performance d'improvisation), les joueuses sont connectées à ce qui se passe autour d'elles. Il ne s'agit pas seulement d'un jeu à deux puisque les autres femmes de la communauté qui les entourent attendent d'elles un jeu digne, et que la gagnante se distingue par son mérite, sa créativité, "sa capacité à surpasser les autres joueuses sur tous les plans, physiques ou esthétiques» (Beaudry 1978, p. 41). Lors de nos entretiens avec Tagaq, l'artiste a fait plusieurs fois mention de l'importance de l'auditoire sur sa performance, "pour la nourrir, pour ressentir sa présence, ses énergies». Or, pour les expérimentations effectuées en janvier 2016, au LARC, nous étions tous réunis dans un environnement contrôlé pour tester le système de captation. L’artiste était donc seule, avec les seuls analystes comme spectateurs, ce qui a permis de

10 Rappelons que, dans notre projet «Development of an interactive prototype of the improvised musical Performance», nous avons utilisé une approche mixte où le quantitatif est au service du qualitatif, en combinant des méthodes provenant de cueillette et d'analyse de données à la fois qualitative (ethnographique, artistique et musicologique) et quantitative (par système de captation optoélectronique VICON qui a permis d'enregistrer les positions tridimensionnelles des marqueurs anatomiques fixés sur le corps de l'artiste). 
mettre en relief l'importance de l'interaction avec le public (absent ce jour-là puisqu'il s'agissait de tester le système), et dont les comportements (émotifs, verbaux, énergétiques) guident Tagaq dans sa façon de raconter son histoire, de communiquer avec le public et en même temps de vivre l'instant présent. Il importe à Tagaq d'instaurer une proximité avec son public.

Les gestes vocaux et les mouvements corporels de l'artiste inuit lors de ses performances n'existent donc pas indépendamment de leur contexte d'énonciation. Les rapports voix/gestes se caractérisent, d'une part, par des imitations par le corps de la voix, ou des compléments apportés par le corps à la production vocale et, d'autre part, par des associations ou des alternances (ils ne débutent pas toujours ensemble, l'un peut s'arrêter tandis que l'autre continue). Il en résulte une variabilité, dont l'analyse suggère que la nature des relations entre les gestes corporels et les gestes vocaux de Tagaq n'en est sûrement pas une d'identité, dans le sens où ils ne feraient qu'un, mais plutôt une interaction: ils se répondent, s'associent, se combinent. Ils existent et se meuvent indépendamment l'un de l'autre, mais se connectent et font du sens ensemble: ils interagissent pour raconter, évoquer l'imaginaire de l'artiste de manière indissociable avec l'expérience vécue dans un espace-temps précis, faisant de ce moment un événement unique. C'est ainsi que le corps et la voix de Tanya Tagaq s'incarnent dans un ensemble expressif de large empan: un acte "performanciel multiplié», selon l'angle d'analyse développé par le musicologue Serge Lacasse (2006, p. 12), c'est-à-dire tout élément de la pratique vocale et instrumentale lié à la production sonore. Pourrait désormais s'ajouter à cette définition la prise en compte des relations entre les gestes vocaux et corporels, en tant que procédés performanciels à part entière. En effet, on ne peut pas comprendre les performances d'improvisation de Tanya Tagaq en les détachant de ses parties: elles sont interconnectées, agissent ensemble et constituent un tout. Un spectacle de Tagaq est donc, en soi, un acte performanciel: elle chante sa vision du katajjaq avec des références claires à la tradition; elle raconte par les gestes de son corps interagissant avec ceux de sa voix son patrimoine inuit et cosmopolite, son engagement social, son combat contre les stéréotypes; elle évoque l'imaginaire du Nord - l'hiver, le froid, les sons glaçants de l'Arctique, sa noirceur, la faune, la cruauté des animaux, leur douceur aussi, leur beauté -, qui prend tout son sens lors des interactions avec le public et les autres musiciens sur scène qui improvisent avec elle: «Quand je suis avec le public, je chante pour donner une voix à ce qui se trouve autour de moi [...] j'observe, je ressens les énergies et les vis dans l'instant présent. Mon corps est totalement mobilisé [...] il devient un catalyseur de ce flux de conscience [stream of consciousness $]^{1{ }^{1}}$ ».

\section{MÉDIAGRAPHIE}

Beaudry, N. 1978. «Le katajjaq un jeu inuit traditionnel », Études/Inuit/Studies, vol. $2, \mathrm{n}^{\mathrm{O}} 1: 35-53$

11 Tanya Tagaq, entretien en autoconfrontation croisée avec Sophie Stévance, avec la collaboration de Serge Lacasse, Michael Garancher et Francis Dubé, Laboratoire de recherche-création en musique et multimédia (LARCEM), Université Laval, 18 juin 2017. 
Clot, Y., et al. 2000. «Entretiens en autoconfrontation croisée: une méthode en clinique de l'activité». Perspectives interdisciplinaires sur le travail et la santé, Vol. 1, $\mathrm{n}^{\circ}{ }_{2}$ : https://pistes.revues.org/3833

Lacasse, S. 2006. «Stratégies narratives dans 'Stan' d'Eminem: le rôle de la voix et de la technologie dans l'articulation du récit phonographique», Protée, vol. $34, \mathrm{n}^{\circ} 2-3$ : 11-26.

Levinson J. 2005. "Contextualisme esthétique», Questions d'interprétation, Vol. $32, \mathrm{n}^{\mathrm{O}} 1$, Printemps: $125-133$

Ruwet, N. 1972. Langue, musique, poésie, Seuil.

Stévance, S., Lacasse, S. 2017. Research-Creation in Music and the Arts: Towards a Collaborative Interdiscipline, Routledge.

Stévance, S., Lacasse, S. 2015. "Research-creation in music as a Collaborative space between musicologist, researchers-creators, and musicians", $\mathrm{Me}$ dia- $N$, Journal of the New Media Caucus, vol. 11, $\mathrm{n}^{\circ} 3$, november: http:// median.newmediacaucus.org/research-creation-explorations/ research-creation-in-music-as-a-collaborative-space/

Stévance, S. 2017a. "From Throat Singing to Transcultural Expression: Tanya Tagaq's Katajjaq Musical 'Signature”, Popular Music in a Transcultural Context, Stan Hawkins (dir.), London: Routledge, 40-61.

2017b. «La recherche-création au carrefour des humanités numériques: capter les mouvements sur scène de Tanya Tagaq", Forum de l'Alliance culture+numérique, 12 octobre 2017, Musée national des beauxarts du Québec (MNBAQ). http://allianceculturenumerique.org/forum/ FORUM Programme-detaille.pdf

. 2016. «La dimensione del «gioco» di gola inuit secondo Tanya Tagaq nella tradizione della nouvelle vocalité occidentale», Le dimensioni della voce, Gervasi, F. (dir.), Besa Editrice, Nardò, 185-199.

.2012. Musique actuelle, Presses de l'Université de Montréal.

2015. "Les performances d'improvisation de Tanya Tagaq: une analyse descriptive de la culture ethno-pop», déc. Récits de société, Revues.org. https://itineraires.revues.org/ 2765

2011. "The Inuit Katajjaq in Popular Culture: The Canadian ThroatSinger Superstar Tanya Tagaq", Itamar. Revista de Investigación Musical: Territorios para el Arte, $\mathrm{n}^{\circ} 3: 79-85$.

Zbikowski, L. 2011, "Musical Gesture and Musical Grammar: A Cognitive Approach", dans A. Gritten et E. King (éd.), New Perspectives on Music and Gesture, Farnham: Ashgate: 83-98.

\section{RÉSUMÉ}

La pratique artistique de Tanya Tagaq se caractérise par le recours à de nombreux effets vocaux, issus tant du katajjaq que d'autres traditions musicales, que la chanteuse semble coordonner à des mouvements et des gestes expressifs. L'hypothèse est que les mouvements du bras, des mains, du corps de Tagaq sur scène possèdent, tout comme les sons qu'elle produit, une dimension symbolique pertinente pour la performance et se coordonnent dans une même expression. Peut-on identifier une typologie 
de correspondances voix/gestes lors d'une performance de Tagaq? Pour explorer son geste musical en rapport avec la production du son, les données ont été générées par la captation des mouvements et de la voix lors de performances scéniques (LARC et Palais Montcalm, Québec, janvier-février 2016), à partir d'un système de motion capture (VICON), d'un microphone de gorge et d'un traitement informatique. On explorera ici un terrain symbolique, éclaté, souvent inaccessible car métaphorique, et peu foulé par la recherche, notamment du fait de la singularité de Tagaq qui intègre autant des éléments de sa culture inuit que ceux de la culture transnationale. Étant donné sa signification expressive pour l'auditoire, il s'agit de saisir ce que pourrait signifier ou exprimer le mouvement corporel en lien avec les émissions vocales de l'artiste.

\section{ABSTRACT}

Tanya Tagaq's artistic work is characterized by the use of vocal effects, borrowed from katajjaq as well as from other musical traditions, which the singer seems to associate with expressive movements and gestures. It is hypothesized that her arm, hands and body movements on stage bear, as it is the case for the sounds she produces, a symbolic dimension relevant to the performance, and associate to create a unified expression. Is it possible to identify a typology of those correspondences between voice and gesture in Tagaq's performance? In order to explore her musical gestures related to her sound production, data have been generated by movement and voice capture during stage performances (LARC and Palais Montcalm palace, Quebec, in January-February 2016), thanks to a motion capture system (VICON), a throat microphone, and information technology processing. In this essay, we explore a symbolic and fragmented field, often unreachable due to its metaphorical aspect, and hitherto rarely researched, a fact also due to the peculiarity of Tagaq's work in integrating simultaneously elements of her Inuit and transnational culture. Given its expressive impact for her audience, our goal is to grasp the meaning and significance of her body movements related to her vocal productions.

\section{BIOGRAPHIE}

Titulaire de la Chaire de recherche du Canada en recherche-création en musique (http:// www.chairs-chaires.gc.ca/chairholders-titulaires/profile eng.aspx?profileId=3481), Sophie Stévance (http://ulaval.academia.edu/SophieStévance) est altiste et artiste lyrique de formation, Professeur agrégée de musicologie, directrice du site Université Laval de l'Observatoire interdisciplinaire de création et de recherche en musique (OICRMUL), du Laboratoire de recherche-création en musique et multimédia (LARCEM), du Groupe de recherche-création en musique (www.grecem.oicrm.org) à la Faculté de musique de l'Université Laval, et rédactrice en chef d'Intersections: revue canadienne de musique. Elle se consacre à l'étude des musiques expérimentales et actuelle(s), plus particulièrement à la pratique contemporaine du chant de gorge par l'artiste Tanya Tagaq, le tout dans une perspective de recherche-création. Elle est l'auteur de plusieurs livres, incluant Pour une éthique partagée de la recherche-création en milieu universitaire (avec S. Lacasse, 2019), Research-Creation in Music and the Arts: Towards a Collaborative Interdiscipline (avec Lacasse, 2017), Quand la musique prend corps (avec M. Desroches 
et S. Lacasse, 2014), Les enjeux de la recherche-création en musique (avec Lacasse, 2013), Musique actuelle (2011), Composer au XXI e siècle (2010), Duchamp, Compositeur (2009) ou Tessier, L'Itinéraire du timbre (2006). Elle a reçu deux prix de l'Académie CharlesCros (2006 et 2010), était finaliste pour le Prix Opus 2014 et 2015 et est récipiendaire de nombreuses subventions de recherche (Conseil de recherche en sciences humaines du Canada, Fonds de recherche québécois, Fondation Canadienne pour l'Innovation/ Fonds des Leaders). Elle poursuit en outre des activités de création et de recherchecréation dans le domaine opératique et la réalisation phonographique. 